\title{
An efficient sequential reaction process to polysubstituted indolizidines and quinolizidines and its application to the total synthesis of indolizidine 223A
}

Wei Zhu, Dapeng Dong, Xiaotao Pu and Dawei Ma*

State Key Laboratory of Bioorganic and Natural Products Chemistry,

Shanghai Institute of Organic Chemistry, Chinese Academy of Sciences,

354 Fenglin Lu, Shanghai 200032, China

Supporting information 


\section{Table of Contents}

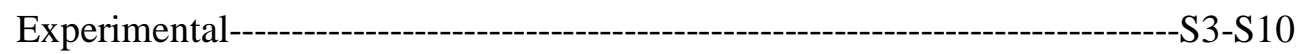

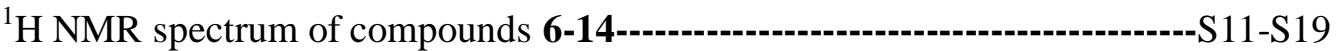

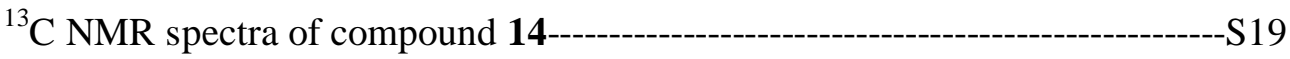




\section{Experimental}

General procedure for table 1: A mixture of 1 (0.22 mmol), 5 (0.23 mmol), anhydrous $\mathrm{K}_{2} \mathrm{CO}_{3}(0.7 \mathrm{mmol})$ and $4 \AA \mathrm{MS}(40 \mathrm{mg})$ in $3 \mathrm{~mL}$ of $\mathrm{MeCN}$ was refluxed until the starting materials disappeared monitored by TLC. The cooled solution was concentrated and partitioned between brine and ether. The organic phase was concentrated and the residue was chromatographed eluting with 1:1 to $1: 10$ ethyl acetate/petroleum ether to afford $\mathbf{6}$.

1,2,3,5,6,7-Hexahydroindolizine-8-carboxylic acid ethyl ester $6 a^{[1]}$ : ${ }^{1} \mathrm{H}$ NMR $\left(\mathrm{CDCl}_{3}, 300 \mathrm{MHz}\right) \delta 4.11(\mathrm{q}, J=7.2 \mathrm{~Hz}, 2 \mathrm{H}), 3.28(\mathrm{t}, J=7.1 \mathrm{~Hz}, 2 \mathrm{H}), 3.14(\mathrm{t}, J=5.8$ $\mathrm{Hz}, 2 \mathrm{H}), 3.05(\mathrm{t}, J=7.8 \mathrm{~Hz}, 2 \mathrm{H}), 2.35(\mathrm{t}, J=6.5 \mathrm{~Hz}, 2 \mathrm{H}), 1.94-1.81(\mathrm{~m}, 4 \mathrm{H}), 1.25(\mathrm{t}$, $J=7.2 \mathrm{~Hz}, 3 \mathrm{H}) ; \mathrm{MS} m / z 195\left(\mathbf{M}^{+}\right)$

8-(Toluene-4-sulfonyl)-1,2,3,5,6,7-hexahydroindolizine 6b: IR (film) 2847, 1592 $\mathrm{cm}^{-1} ;{ }^{1} \mathrm{H} \mathrm{NMR}\left(\mathrm{CDCl}_{3}, 300 \mathrm{MHz}\right) \delta$ 7.72-7.68 (m, 2H), 7.28-7.23 (m, 2H), $3.28(\mathrm{t}, J=$ $7.2 \mathrm{~Hz}, 2 \mathrm{H}), 3.14-3.09(\mathrm{~m}, 4 \mathrm{H}), 2.40(\mathrm{~s} .3 \mathrm{H}), 2.33(\mathrm{t}, J=6.0 \mathrm{~Hz}, 2 \mathrm{H}), 1.98-1.88(\mathrm{~m}$, 2H), 1.86-1.78 (m, 2H); MS m/z $277\left(\mathrm{M}^{+}\right)$; HRMS calcd for $\mathrm{C}_{15} \mathrm{H}_{19} \mathrm{NSO}_{2} 277.1180$ $\left(\mathrm{M}^{+}\right)$, found 277.1158.

(1,2,3,5,6,7-Hexahydroindolizin-8-yl)phosphonic acid diethyl ester 6c: IR (film) $2979,1610,1238 \mathrm{~cm}^{-1} ;{ }^{1} \mathrm{H} \mathrm{NMR}\left(\mathrm{CDCl}_{3}, 300 \mathrm{MHz}\right) \delta 4.04-3.90(\mathrm{~m}, 4 \mathrm{H}), 3.22(\mathrm{t}, J=$ $6.9 \mathrm{~Hz}, 2 \mathrm{H}), 3.15(\mathrm{t}, J=5.8 \mathrm{~Hz}, 2 \mathrm{H}), 2.90(\mathrm{t}, J=7.4 \mathrm{~Hz}, 2 \mathrm{H}), 2.18(\mathrm{q}, J=6.1 \mathrm{~Hz}, 2 \mathrm{H})$, 
1.92-1.81 (m, 4H), $1.29(\mathrm{t}, J=7.2 \mathrm{~Hz}, 6 \mathrm{H}) ; \mathrm{MS} \mathrm{m} / z 259\left(\mathrm{M}^{+}\right)$; HRMS calcd for $\mathrm{C}_{12} \mathrm{H}_{23} \mathrm{NPO}_{3} .260 .1410(\mathrm{M}+\mathrm{H})^{+}$, found 260.1415.

3,4,6,7,8,9-Hexahydro-2H-quinolizine-1-carboxylic acid ethyl ester $6 \mathrm{~d}^{[1]}:{ }^{1} \mathrm{H}$ NMR $\left(\mathrm{CDCl}_{3}, 300 \mathrm{MHz}\right) \delta 4.08(\mathrm{q}, J=7.2 \mathrm{~Hz}, 2 \mathrm{H}), 3.13-3.06(\mathrm{~m}, 4 \mathrm{H}), 3.01(\mathrm{t}, J=6.4 \mathrm{~Hz}$ 2H), $2.38(\mathrm{t}, J=12.9 \mathrm{~Hz}, 2 \mathrm{H}), 1.80-1.73(\mathrm{~m}, 4 \mathrm{H}), 1.63-1.59(\mathrm{~m}, 2 \mathrm{H}), 1.24(\mathrm{t}, J=7.2$ $\mathrm{Hz}, 3 \mathrm{H}) ; \mathrm{MS} m / z 209\left(\mathrm{M}^{+}\right)$.

9-(Toluene-4-sulfonyl)-1,3,4,6,7,8-hexahydro-2H-quinolizine 6e: IR (film) 2948, 1558, $1270 \mathrm{~cm}^{-1} ;{ }^{1} \mathrm{H}$ NMR $\left(\mathrm{CDCl}_{3}, 300 \mathrm{MHz}\right) \delta$ 7.71-7.68 (m, 2H), 7.27-7.24 (m, 2H), 3.12-3.04 (m, 4H), $2.84(\mathrm{t}, J=6.5 \mathrm{~Hz}, 2 \mathrm{H}), 2.53(\mathrm{t}, J=6.2 \mathrm{~Hz}, 2 \mathrm{H}), 2.40(\mathrm{~s}, 3 \mathrm{H})$, 1.83-1.77 (m, 2H), 1.74-1.68 (m, 2H), 1.60-1.55 (m, 2H); MS m/z $291\left(\mathrm{M}^{+}\right)$; HRMS calcd for $\mathrm{C}_{16} \mathrm{H}_{21} \mathrm{NSO}_{2} 291.1315\left(\mathrm{M}^{+}\right)$, found 291.1304 .

(3,4,6,7,8,9-Hexahydro-2H-quinolizin-1-yl)phosphonic acid diethyl ester 6f: IR (film) 2939, 1577. $1235 \mathrm{~cm}^{-1} ;{ }^{1} \mathrm{H}$ NMR $\left(\mathrm{CDCl}_{3}, 300 \mathrm{MHz}\right) \delta$ 4.06-3.96 (m, 4H), 3.07-3.01 (m, 4H), 2.80 (br s, 2H), 2.27-2.21 (m, 2H), 1.85-1.73 (m, 4H), 1.64-1.58 (m, 2H), 1.34-1.28 (m, 6H); MS m/z $273\left(\mathrm{M}^{+}\right)$; HRMS calcd for $\mathrm{C}_{13} \mathrm{H}_{25} \mathrm{NPO}_{3} 274.1567(\mathrm{M}$ $+\mathrm{H})^{+}$, found 274.1565 .

2,3,4,6,7,8,9,10-Octahydropyrido[1,2-a]azepine-1-carboxylic acid ethyl ester $6 \mathrm{~g}^{[1]}$ :

${ }^{1} \mathrm{H}$ NMR $\left(\mathrm{CDCl}_{3}, 300 \mathrm{MHz}\right) \delta 4.10(\mathrm{q}, J=7.2 \mathrm{~Hz}, 2 \mathrm{H}), 3.37-3.34(\mathrm{~m}, 2 \mathrm{H}), 3.21(\mathrm{t}, J=$ $5.8 \mathrm{~Hz}, 2 \mathrm{H}), 3.15(\mathrm{t}, J=4.7 \mathrm{~Hz}, 2 \mathrm{H}), 2.37(\mathrm{t}, J=6.3 \mathrm{~Hz}, 2 \mathrm{H}), 1.81-1.77(\mathrm{~m}, 2 \mathrm{H})$, 
1.65-1.57 (m, 6H), $1.25(\mathrm{t}, J=7.2 \mathrm{~Hz}, 3 \mathrm{H}) ; \mathrm{MS} \mathrm{m} / z 223\left(\mathrm{M}^{+}\right)$.

(2R)-3,4,6,7,8,9-Hexahydro-2H-quinolizine-1-carboxylic acid ethyl ester $6 \mathrm{~h}^{[2]}$ : $[\alpha]_{\mathrm{D}}^{30}-1.05$ (c 1.2, EtOH); IR (film) 2932, 1680, 1593, $1282 \mathrm{~cm}^{-1} ;{ }^{1} \mathrm{H}$ NMR $\left(\mathrm{CDCl}_{3}\right.$, $300 \mathrm{MHz}) \delta 4.11(\mathrm{q}, J=7.2 \mathrm{~Hz}, 2 \mathrm{H}), 3.51(\mathrm{dt}, J=9.3,7.0 \mathrm{~Hz}, 1 \mathrm{H}), 3.24-3.14(\mathrm{~m}, 2 \mathrm{H})$, 3.10-3.04 (m, 2H), $2.44(\mathrm{dt}, J=15.8,5.1 \mathrm{~Hz}, 1 \mathrm{H}), 2.27-2.16(\mathrm{~m}, 1 \mathrm{H}), 1.97-1.85(\mathrm{~m}$, 2H), 1.86-1.75 (m, 2H), 1.68-1.55 (m, 2H), 1.38-1.23 (m, 1H), 0.89 (t, J=7.2 Hz, 3H); MS m/z $265\left(\mathrm{M}^{+}\right)$; HRMS calcd for $\mathrm{C}_{16} \mathrm{H}_{29} \mathrm{NO}_{2}$ 266.2115, found 266.2119.

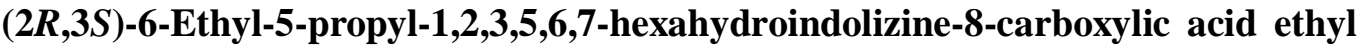
ester 6i: $[\alpha]_{D}{ }^{17}+16.1\left(c 1.2, \mathrm{CHCl}_{3}\right)$; IR (film) $1678,1593 \mathrm{~cm}^{-1} ;{ }^{1} \mathrm{H}$ NMR $\left(\mathrm{CDCl}_{3}, 300\right.$ MHz) $\delta 4.11(\mathrm{q}, J=7.2 \mathrm{~Hz}, 2 \mathrm{H}), 3.56(\mathrm{dt}, J=9.3,7.1 \mathrm{~Hz}, 1 \mathrm{H}), 3.24-3.17(\mathrm{~m}, 1 \mathrm{H})$, 3.09-3.01 (m, 3H), 2.39-2.19 (m, 2H), 1.97-1.86 (m, 2H), $1.65(\mathrm{~m}, 1 \mathrm{H}), 1.53(\mathrm{~m}, 1 \mathrm{H})$, $1.40-1.23(\mathrm{~m}, 6 \mathrm{H}), 1.17-1.10(\mathrm{~m}, 2 \mathrm{H}), 0.95-0.86(\mathrm{~m}, 6 \mathrm{H}) ;{ }^{13} \mathrm{C} \mathrm{NMR}\left(\mathrm{CDCl}_{3}, 75 \mathrm{MHz}\right)$ $\delta 169.3,157.2,84.6,58.3,58.0,52.4,35.4,34.7,32.7,25.3,22.3,21.2,19.2,14.8,14.1$ 12.0; MS m/z $265\left(\mathrm{M}^{+}\right)$; HRMS calcd for $\mathrm{C}_{16} \mathrm{H}_{28} \mathrm{NO}_{2} 266.2115(\mathrm{M}+\mathrm{H})^{+}$, found 266.2113.

$(2 R, 3 S)-3-E t h y l-4-p r o p y l-3,4,6,7,8,9-h e x a h y d r o-2 H$-quinolizine-1-carboxylic acid ethyl ester $6 \mathbf{j}:[\alpha]_{D}{ }^{18}-139.2\left(c 0.5, \mathrm{CHCl}_{3}\right)$; IR (film) $1730,1673,1556 \mathrm{~cm}^{-1} ;{ }^{1} \mathrm{H}$ NMR $\left(\mathrm{CDCl}_{3}, 300 \mathrm{MHz}\right) \delta 4.08(\mathrm{q}, J=7.0 \mathrm{~Hz}, 2 \mathrm{H}), 3.35-3.10(\mathrm{~m}, 3 \mathrm{H}), 2.90-2.81(\mathrm{~m}, 2 \mathrm{H})$, 2.37-2.23 (m, 2H), 1.74-1.64 (m, 3H), 1.57-1.52 (m, 4H), 1.34-1.16 (m, 7H), 0.94-0.88 $(\mathrm{m}, 6 \mathrm{H}) ; \mathrm{MS} m / 2,265\left(\mathrm{M}^{+}\right)$; HRMS calcd for $\mathrm{C}_{17} \mathrm{H}_{30} \mathrm{NO}_{2} 280.2271(\mathrm{M}+\mathrm{H})^{+}$, found 
280.2277.

(2S)-4-(3,4-Dimethoxyphenyl)-3,4,6,7,8,9-hexahydro-2H-quinolizine-1-carboxylic acid ethyl ester 6k: $[\alpha]_{\mathrm{D}}{ }^{19}+44.3\left(c 1.4, \mathrm{CHCl}_{3}\right)$; IR (film) 2939, $1669,1549 \mathrm{~cm}^{-1} ;{ }^{1} \mathrm{H}$ NMR $\left(\mathrm{CDCl}_{3}, 300 \mathrm{MHz}\right) \delta$ 6.83-6.81 (m, 1H), 6.72-6.69 (m, 2H), 4.20 (br s, 1H), 4.11-4.06 (m, 2H), 3.87 (s, 3H), $3.86(\mathrm{~s}, 3 \mathrm{H}), 3.21-3.15(\mathrm{~m}, 4 \mathrm{H}), 2.57-2.45(\mathrm{~m}, 1 \mathrm{H})$, 2.10-1.69 (m, 7H), $1.24(\mathrm{t}, J=7.2 \mathrm{~Hz}, 3 \mathrm{H}) ;{ }^{13} \mathrm{C} \mathrm{NMR}\left(\mathrm{CDCl}_{3}, 75 \mathrm{MHz}\right) \delta 168.8,156.4$, $148.9,147.9,135.1,118.3,111.0,109.3,91.5,62.9,58.5,55.9,55.8,49.6,28.6,27.6$, 23.4, 20.4, 19.2, 14.7; ESI-MS $m / z 346(\mathrm{M}+\mathrm{H})^{+}$; HRMS calcd for $\mathrm{C}_{20} \mathrm{H}_{28} \mathrm{NO}_{4}$ 346.2013 $(\mathrm{M}+\mathrm{H})^{+}$, found 346.2013.

(2S)-[4-(3,4-Dimethoxyphenyl)-3,4,6,7,8,9-hexahydro-2H-quinolizin-1-yl]phenyl-

methanone 6n: $[\alpha]_{\mathrm{D}}{ }^{21}+144.9\left(c 1.2, \mathrm{CHCl}_{3}\right)$; IR (film) 2933, 1712, 1595, $1516 \mathrm{~cm}^{-1}$; ${ }^{1} \mathrm{H}$ NMR $\left(\mathrm{CDCl}_{3}, 300 \mathrm{MHz}\right)$ 6 7.49-7.46 (m, 2H), 7.40-7.30 (m, 3H), 6.89-6.86 (m, 1H), 6.79-6.74 (m, 2H), $4.29(\mathrm{t}, J=3.9 \mathrm{~Hz}, 1 \mathrm{H}), 3.88(\mathrm{~s}, 3 \mathrm{H}), 3.87(\mathrm{~s}, 3 \mathrm{H}), 3.29-3.00$ (m, 4H), 2.32-2.19 (m, 2H), 2.03-1.96 (m, 1H), 1.88-1.64 (m, 5H); ${ }^{13} \mathrm{C} \mathrm{NMR}\left(\mathrm{CDCl}_{3,}\right.$, $75 \mathrm{MHz}) \delta 194.7,158.6,149.1,148.2,143.9,135.0,129.3$ (2C), 127.9 (2C), 127.6 (2C), 118.4, 111.1, 109.3, 102.5, 63.5, 55.9, 49.5, 29.4, 28.8, 23.2, 22.6, 20.4; ESI-HRMS calcd. for $\mathrm{C}_{24} \mathrm{H}_{28} \mathrm{NO}_{3} 378.2070(\mathrm{M}+\mathrm{H})^{+}$, found 378.2064.

\section{(2S,3R,1'R)-3-[Benzyl-(1-phenylethyl)amino]-2-ethylhexanoic acid tert-butyl}

ester 8. To a stirred solution of (+)- $N$-benzyl- $\alpha$-methylbenzylamine (10.5 g, $50 \mathrm{mmol})$ in $30 \mathrm{~mL}$ of dry toluene was slowly added $n$-BuLi $(1.6 \mathrm{M}, 31 \mathrm{~mL})$ at $0{ }^{\circ} \mathrm{C}$. The 
resultant solution was stirred for $1 \mathrm{~h}$ before been cooled to $-78{ }^{\circ} \mathrm{C}$. Then tert-butyl 2-ethyl-2-hexenoate $(3.96 \mathrm{~g}, 20 \mathrm{mmol})$ in $20 \mathrm{~mL}$ dry toluene was added slowly. The mixture was stirred for $1 \mathrm{~h}$ at $-78{ }^{\circ} \mathrm{C}$, then warmed to $-40{ }^{\circ} \mathrm{C}$ over $1 \mathrm{~h}$ and maintained for $1 \mathrm{~h}$ before it was recooled to $-78{ }^{\circ} \mathrm{C}$. After precooled THF $\left(-78{ }^{\circ} \mathrm{C}, 200 \mathrm{~mL}\right)$ was added quickly, the solution was stirred for another $30 \mathrm{~min}$ before a precooled solution of 2,6-di-tert-butyl-4-methylphenol $(18 \mathrm{~g}, 100 \mathrm{mmol})$ in THF $\left(-78{ }^{\circ} \mathrm{C}, 20 \mathrm{~mL}\right)$ was added in one portion. After it was stirred for a further $10 \mathrm{~min}$ at $-78{ }^{\circ} \mathrm{C}$, the solution was warmed to room temperature over $1 \mathrm{~h}$. Evaporation of the mixture left a residue which was partitioned between brine and $\mathrm{Et}_{2} \mathrm{O}$ and the organic layer was dried over $\mathrm{MgSO}_{4}$, removed in vacuum. The residue was purified via chromatograph to give $5.5 \mathrm{~g}$ $(67 \%)$ of $\mathbf{8}$ as a colorless oil. $[\alpha]_{\mathrm{D}}{ }^{15}+43\left(c 1.25, \mathrm{CHCl}_{3}\right)$; IR (film) $2966,2874,1725$, $1455 \mathrm{~cm}^{-1} ;{ }^{1} \mathrm{H} \mathrm{NMR}\left(\mathrm{CDCl}_{3}, 300 \mathrm{MHz}\right) \delta 7.35-7.21(\mathrm{~m}, 10 \mathrm{H}), 3.91(\mathrm{dq}, J=14,3.5 \mathrm{~Hz}$, $3 \mathrm{H}), 2.82(\mathrm{~m}, 1 \mathrm{H}), 2.21(\mathrm{~m}, 1 \mathrm{H}), 1.52-1.23(\mathrm{~m}, 4 \mathrm{H}), 1.41(\mathrm{~s}, 9 \mathrm{H}), 1.26(\mathrm{~d}, J=6.9 \mathrm{~Hz}$ $3 \mathrm{H}), 0.94(\mathrm{~m}, 2 \mathrm{H}), 0.81(\mathrm{t}, J=7.2 \mathrm{~Hz}, 3 \mathrm{H}), 0.66(\mathrm{t}, J=7.2 \mathrm{~Hz}, 3 \mathrm{H}) ;{ }^{13} \mathrm{C} \mathrm{NMR}\left(\mathrm{CDCl}_{3}\right.$, $75 \mathrm{MHz}) \delta 175.1,144.8,142.7,128.2,128.0,127.9,127.8,127.0,126.7,126.3,79.8$ $60.1,51.2,32.7,28.0,24.9,21.1,20.0,14.3,11.9$; EI-HRMS calcd. for $\mathrm{C}_{27} \mathrm{H}_{39} \mathrm{NO}_{2}$ 409.2474 $\left(\mathrm{M}^{+}\right)$, found 409.2455.

(2S,3R,1'R)-3-[Benzyl(1-phenylethyl)amino]-2-ethylhexan-1-ol 9. A mixture of $\mathbf{8}(5.5 \mathrm{~g}, 13.4 \mathrm{mmol})$ and $\mathrm{LiAlH}_{4}(1.5 \mathrm{~g})$ in dry THF (60 mL) was refluxed for $12 \mathrm{~h}$. Then the reaction was quenched with $15 \% \mathrm{NaOH}(1 \mathrm{~mL})$ and filtered. The filtrate was concentrated and purified via chromatography to give 9 as a viscous oil. $[\alpha]_{\mathrm{D}}{ }^{20}-40.6$ (c 1.23, $\left.\mathrm{CHCl}_{3}\right) ;{ }^{1} \mathrm{H} \mathrm{NMR}\left(\mathrm{CDCl}_{3}, 300 \mathrm{MHz}\right) \delta$ 7.44-7.21 (m, 10H), 3.95-3.90 (m, 3H), 
$3.72(\mathrm{~d}, J=13.5 \mathrm{~Hz}, 1 \mathrm{H}), 3.43-3.39(\mathrm{~m}, 1 \mathrm{H}), 3.00(\mathrm{dt}, J=10.2,3.6 \mathrm{~Hz}, 1 \mathrm{H}), 2.94-2.88$

$(\mathrm{m}, 2 \mathrm{H}), 1.72-1.70(\mathrm{~m}, 1 \mathrm{H}), 1.61-1.50(\mathrm{~m}, 2 \mathrm{H}), 1.40-1.22(\mathrm{~m}, 5 \mathrm{H}), 0.97(\mathrm{t}, J=7.1 \mathrm{~Hz}$ $3 \mathrm{H}), 0.86(\mathrm{t}, J=7.4 \mathrm{~Hz}, 3 \mathrm{H})$; ESI-HRMS calcd. for $\mathrm{C}_{23} \mathrm{H}_{34} \mathrm{NO} 340.2635(\mathrm{M}+\mathrm{H})^{+}$, found 340.2639 .

(1R,2S)-2-Chloromethyl-1-propylbutylamine 10. To a stirred solution of 9 (0.45 g, $1.33 \mathrm{mmol})$ in $4 \mathrm{~mL}$ dry $\mathrm{CHCl}_{3}$ at $0{ }^{\circ} \mathrm{C}$ was added slowly $\mathrm{SOCl}_{2}(0.2 \mathrm{~mL}, 2.66 \mathrm{mmol})$. Then the mixture was refluxed for $1 \mathrm{~h}$. Evaporation of the solvent in vacuum gave the desired product as a hydrochloride salt, which was dissolved in $\mathrm{MeOH}(5 \mathrm{~mL})$, and hydrogenated with $20 \% \mathrm{Pd}(\mathrm{OH})_{2} / \mathrm{C}(200 \mathrm{mg})$ at $50 \mathrm{~atm}, 40{ }^{\circ} \mathrm{C}$ for $48 \mathrm{~h}$. The solution was filtered, and the filtrate was concentrated in vacuum to give $\mathbf{1 0}$ as its hydrochloride salt, which was used directly for next step due to its instability.

(2R,3R)-6-Ethyl-5-propyl-1,2,3,5,6,7-hexahydroindolizine-8-carboxylic acid ethyl

ester 11. Following the procedure for Table 1 as mentioned above, 11 was prepared from $1 \mathrm{a}$ and $\mathbf{1 0}$ in $80 \%$ yield. $[\alpha]_{\mathrm{D}}{ }^{18}-40.3\left(c 0.75, \mathrm{CHCl}_{3}\right) ;{ }^{1} \mathrm{H} \mathrm{NMR}\left(\mathrm{CDCl}_{3}, 300 \mathrm{MHz}\right)$ א 4.22-4.04 (m, 2H), 3.60-3.52 (m, 1H), 3.39-3.00 (m, 5H), 2.79-2.77 (m, 1H), 2.06-1.89 (m, 2H), 1.46-1.23 (m, 10H), 0.97-0.91 (m, 6H); ESI m/z $266(\mathrm{M}+\mathrm{H})^{+}$ HRMS calcd for $\mathrm{C}_{16} \mathrm{H}_{28} \mathrm{NO}_{2} 266.2115(\mathrm{M}+\mathrm{H})^{+}$, found 266.2113.

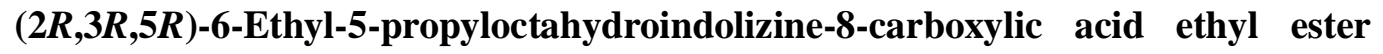
12b. A mixture of $\mathbf{1 1}(40 \mathrm{mg}, 0.15 \mathrm{mmol})$ in glacial acid $(1 \mathrm{ml})$ was hydrogenated over platinum dioxide $(8 \mathrm{mg})$ at room temperature and 1 atm for $2 \mathrm{~h}$. After the volatiles was 
removed, the residue was treated with aqueous $\mathrm{NaHCO}_{3}$, and extracted with $\mathrm{CH}_{2} \mathrm{Cl}_{2}$, dried over $\mathrm{MgSO}_{4}$. The solution was concentrated to give the desired product as a mixture of two diastereoisomers $\mathbf{1 2} \mathbf{a}$ and $\mathbf{1 2} \mathbf{b}$, which was dissolved in anhydrous EtOH containing catalytic amount of $\mathrm{NaOEt}$, and refluxed for $5 \mathrm{~h}$ before it was quenched with aqueous $\mathrm{NaHCO}_{3}$. The aqueous layer was extracted with EtOAc, and dried over $\mathrm{MgSO}_{4}$. The solution was concentrated and the residue was chromatographed on silica gel to afford $32 \mathrm{mg}(81 \%)$ of $\mathbf{1 2 b}$ as a single isomer. $[\alpha]_{\mathrm{D}}{ }^{18}$ -27.3 (c 0.5, $\left.\mathrm{CHCl}_{3}\right)$; IR (film) 2962, 1734, $1261 \mathrm{~cm}^{-1} ;{ }^{1} \mathrm{H} \mathrm{NMR}\left(\mathrm{CDCl}_{3}, 300 \mathrm{MHz}\right)$ א 4.13-4.04 (m, 2H), $3.07(\mathrm{~d}, J=11.4 \mathrm{~Hz}, 1 \mathrm{H}), 2.90(\mathrm{t}, J=8.1 \mathrm{~Hz}, 1 \mathrm{H}), 2.09-1.82(\mathrm{~m}$, 4H), 1.75-1.06 (m, 15H), 0.92-0.86 (m, 6H); ${ }^{13} \mathrm{C} \mathrm{NMR}\left(\mathrm{CDCl}_{3}, 75 \mathrm{MHz}\right) \delta 174.4,67.1$, $60.1,54.5,54.1,50.9,42.3,37.3,32.4,28.9,20.9,19.8,17.7,14.4,14.3,12.8$ ESI-HRMS calcd. for $\mathrm{C}_{16} \mathrm{H}_{29} \mathrm{NO}_{2} 267.2193\left(\mathrm{M}^{+}\right)$, found 267.2190.

Indolizidine 223A 14. A mixture of 12b $(30 \mathrm{mg}, 0.112 \mathrm{mmol})$ and $\mathrm{LiAlH}_{4}(20 \mathrm{mg})$ in dry THF $(1 \mathrm{~mL})$ was stirred at room temperature for $1 \mathrm{~h}$ before it was quenched with $15 \% \mathrm{NaOH}(0.15 \mathrm{~mL})$. The mixture was filtered and the filtrate was dried over $\mathrm{MgSO}_{4}$ and concentrated to give the crude alcohol $\mathbf{1 3}$ (91\% yield), which was used directly in the next step.

To a solution of oxalyl chloride $(0.23 \mathrm{mmol})$ in $1 \mathrm{~mL}$ dry $\mathrm{CH}_{2} \mathrm{Cl}_{2}$ was added dropwise a solution of DMSO $(0.33 \mathrm{mmol})$ in $0.5 \mathrm{~mL}$ of $\mathrm{CH}_{2} \mathrm{Cl}_{2}$ at $-78{ }^{\circ} \mathrm{C}$. After the solution was stirred for $15 \mathrm{~min}$, a solution of the above alcohol in $0.5 \mathrm{~mL}$ of $\mathrm{CH}_{2} \mathrm{Cl}_{2}$ was added slowly, and stirred for another $15 \mathrm{~min}$. After $\mathrm{Et}_{3} \mathrm{~N}(0.56 \mathrm{mmol})$ was added, the stirring was continued for $5 \mathrm{~min}$ and then the solution was allowed to warm to 
room temperature. The mixture was partitioned between water and $\mathrm{CH}_{2} \mathrm{Cl}_{2}$. The combined organic layers were washed with water and dried over $\mathrm{MgSO}_{4}$. Evaporation on vacuum gave the crude aldehyde.

To a solution of $\mathrm{Ph}_{3} \mathrm{P}^{+} \mathrm{CH}_{3} \mathrm{I}^{-}(132 \mathrm{mg}, 0.33 \mathrm{mmol})$ in THF (2 mL) was added NaHMDS $(2.0 \mathrm{M}, 0.15 \mathrm{~mL}, 0.31 \mathrm{mmol})$ at $-78{ }^{\circ} \mathrm{C}$. After $30 \mathrm{~min}$, a solution of the above crude aldehyde in $1 \mathrm{~mL}$ of THF was added. The solution was stirred for $1 \mathrm{~h}$, then warmed up to $0{ }^{\circ} \mathrm{C}$ before water was added to quench the reaction. The mixture was extracted with $\mathrm{Et}_{2} \mathrm{O}$, washed with brine, and dried over $\mathrm{MgSO}_{4}$. Flash chromatography on silica gel gave the crude olefin, which was directly hydrogenated on $10 \% \mathrm{Pd}-\mathrm{C}$ (5 $\mathrm{mg}$ ) in $1 \mathrm{mLEtOAc}$ at $1 \mathrm{~atm}$ and room temperature for $2 \mathrm{~h}$. Removal of the solvent and purification on silica gel gave $11 \mathrm{mg}$ (49\% yield from 13) of Indolizidine $223 \mathrm{~A}$ as a volatile oil. Data for its $\mathrm{DCl}$ salt: $[\alpha]_{\mathrm{D}}{ }^{15}-38.0\left(c 0.30, \mathrm{CHCl}_{3}\right) ;{ }^{1} \mathrm{H} \mathrm{NMR}(300 \mathrm{MHz}$, $\left.\mathrm{D}_{2} \mathrm{O}\right) \delta 3.58(\mathrm{dt}, J=9.6,2.7 \mathrm{~Hz}, 1 \mathrm{H}), 3.15(\mathrm{dt}, J=11.0,3.6 \mathrm{~Hz}, 1 \mathrm{H}), 2.95(\mathrm{q}, J=9.1 \mathrm{~Hz}$, 1H), $2.86(\mathrm{dt}, J=11.5,6.0 \mathrm{~Hz}, 1 \mathrm{H}), 2.28(\mathrm{~m}, 1 \mathrm{H}), 2.05(\mathrm{dd}, J=13.2,2.5 \mathrm{~Hz}, 1 \mathrm{H})$, 1.97-1.88 (m, 3H), 1.74-1.37 (m, 7H), 1.22-1.12 (m, 4H), 0.88-0.80 (m, 9H); ${ }^{13} \mathrm{C}$ NMR $\left(\mathrm{D}_{2} \mathrm{O}, 75 \mathrm{MHz}\right) \delta 73.8,68.1,52.7,36.8,36.9,31.4,31.3,27.7,26.0,20.2,19.3,18.5$ 14.7, 13.0, 11.4; MS-HRMS calcd. for $\mathrm{C}_{15} \mathrm{H}_{29} \mathrm{~N} 223.3976\left(\mathrm{M}^{+}\right)$, found 223.3958. 

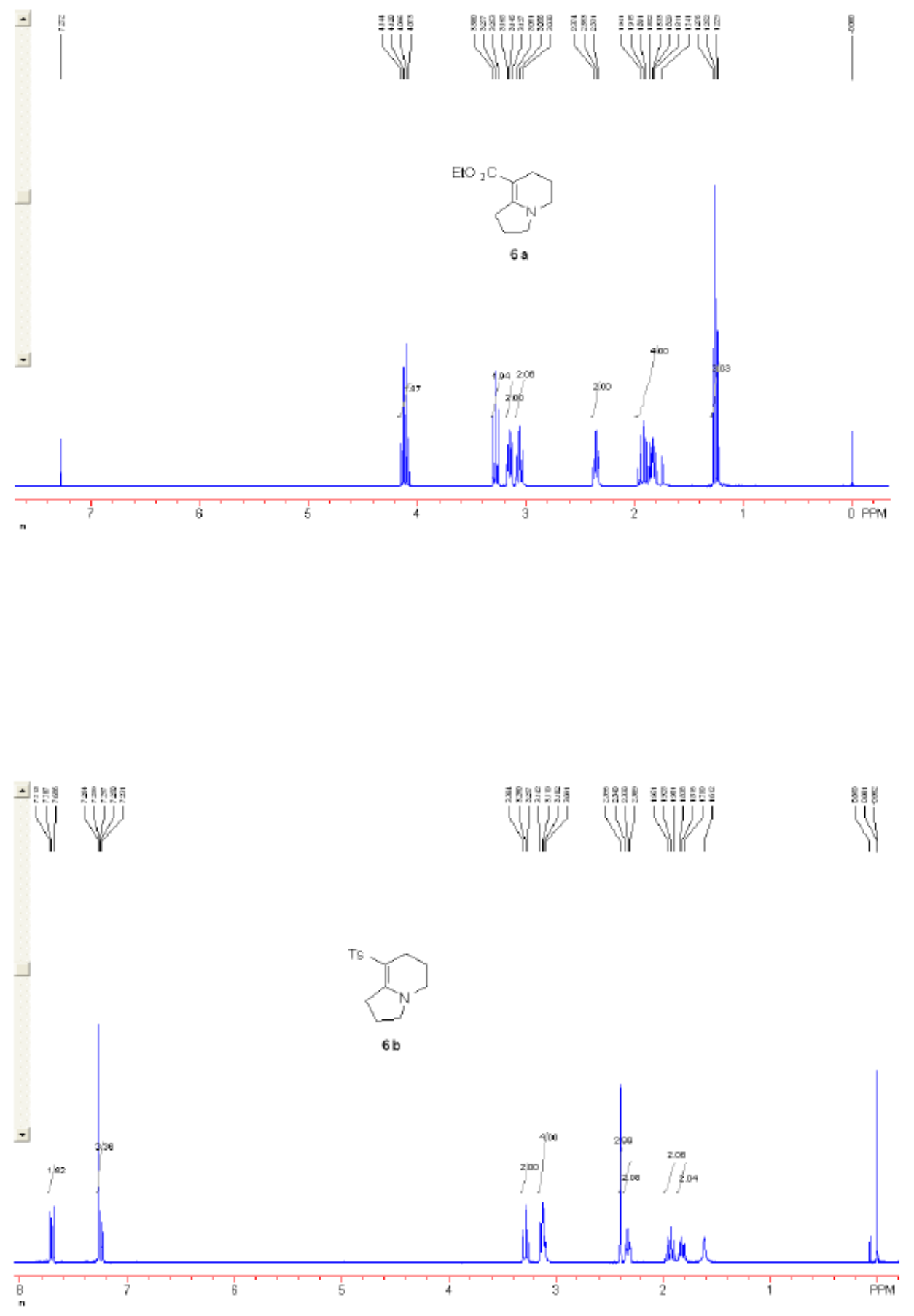

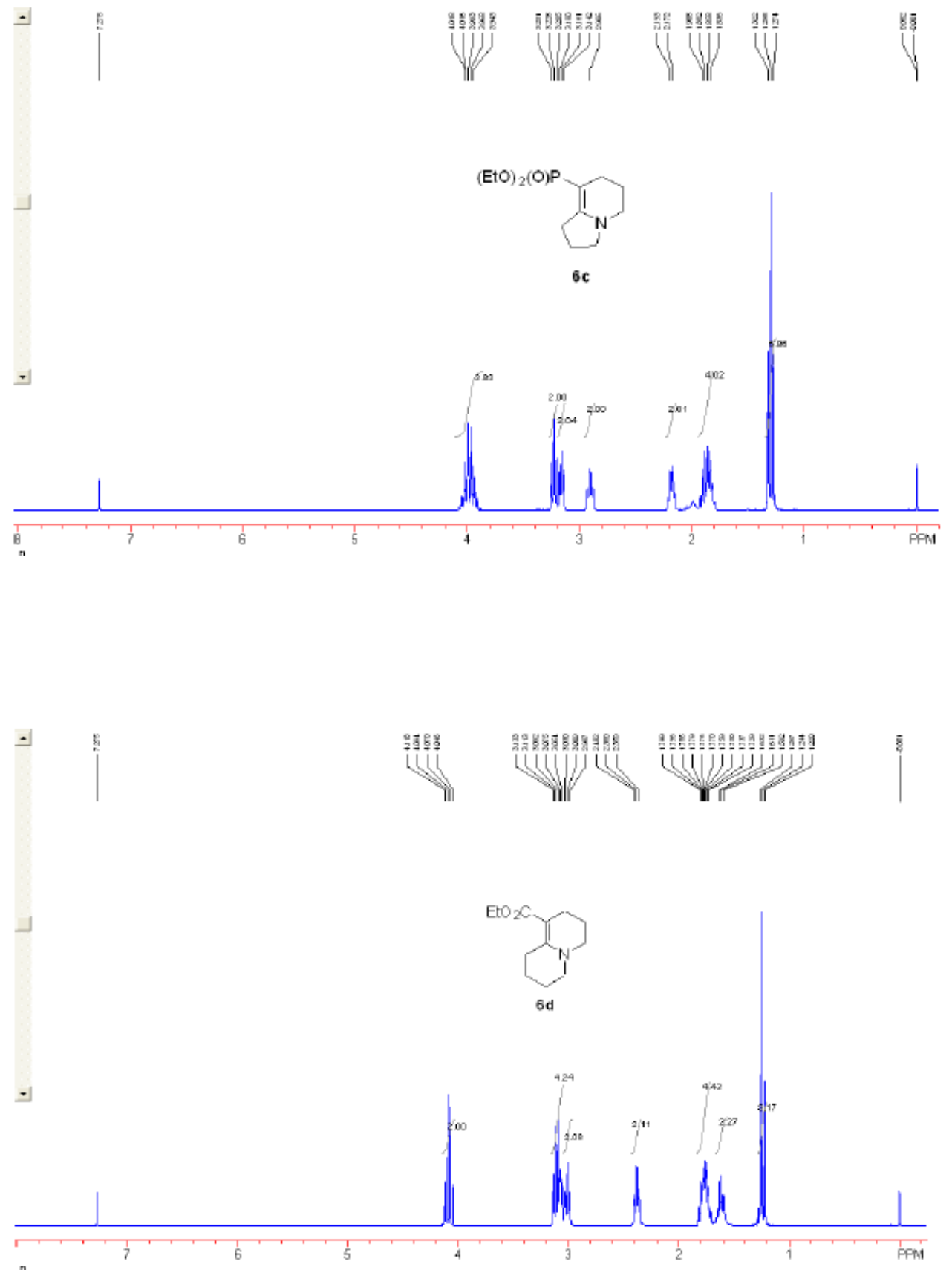


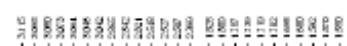

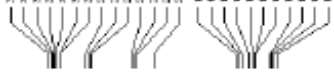

4
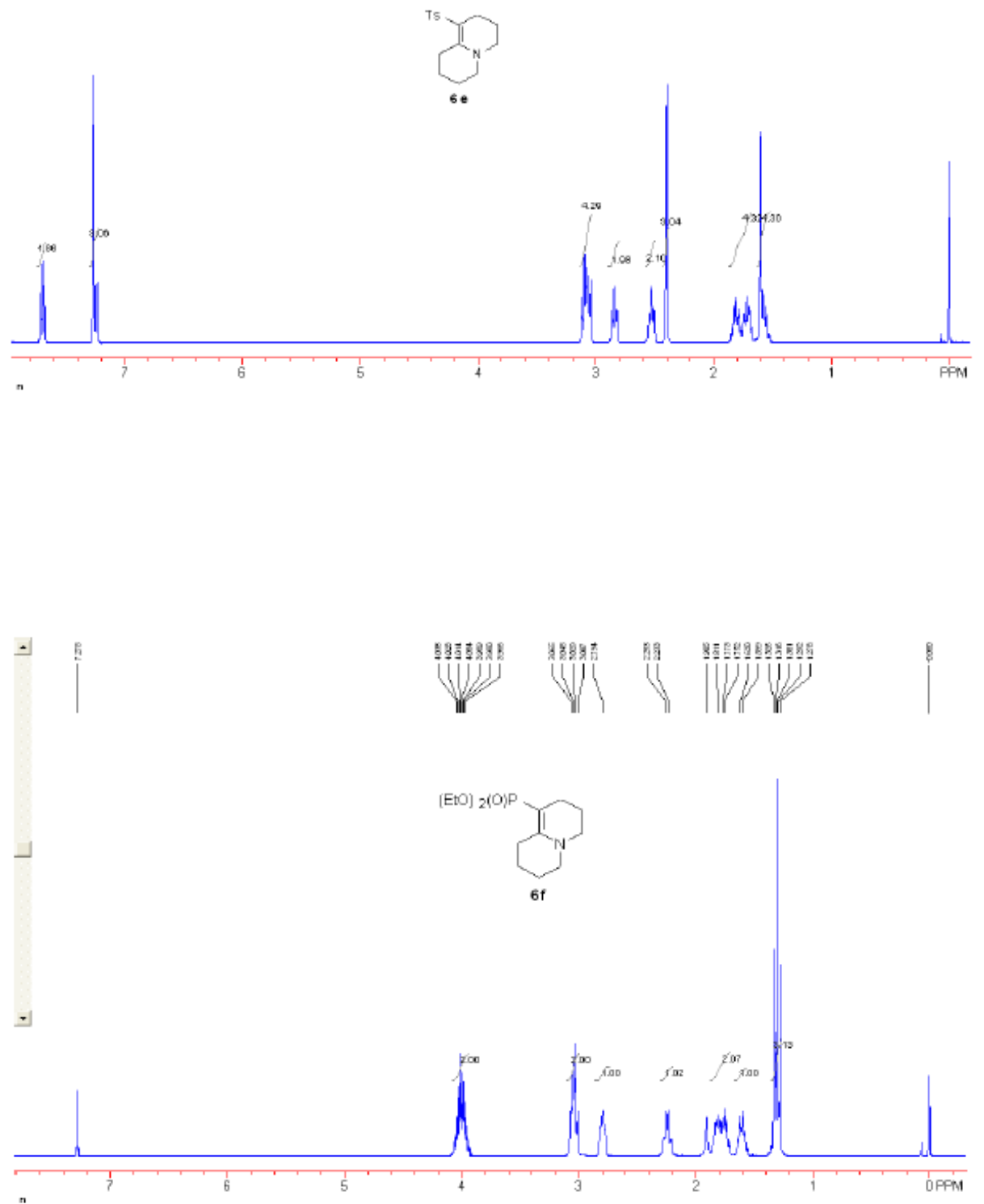

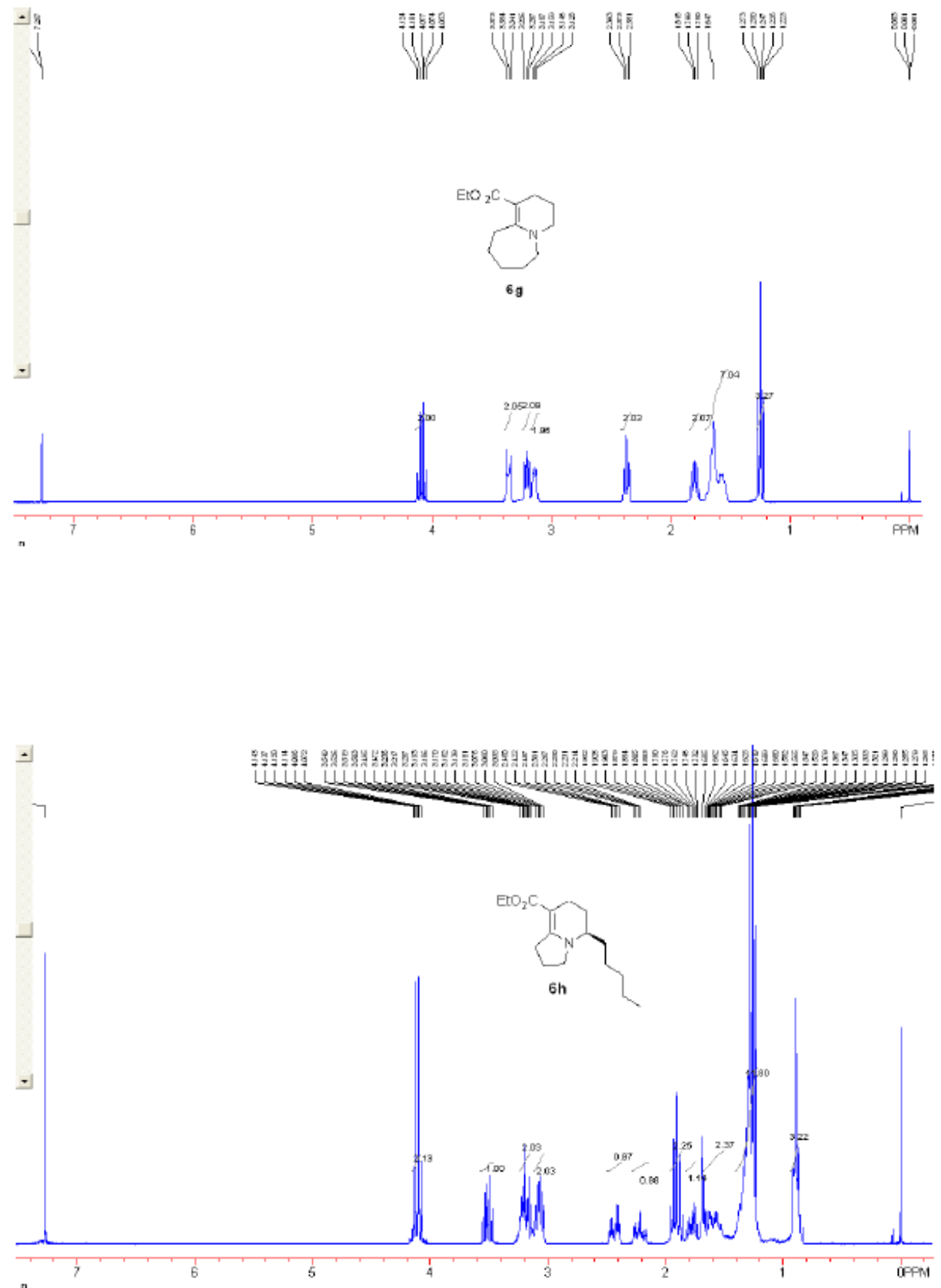

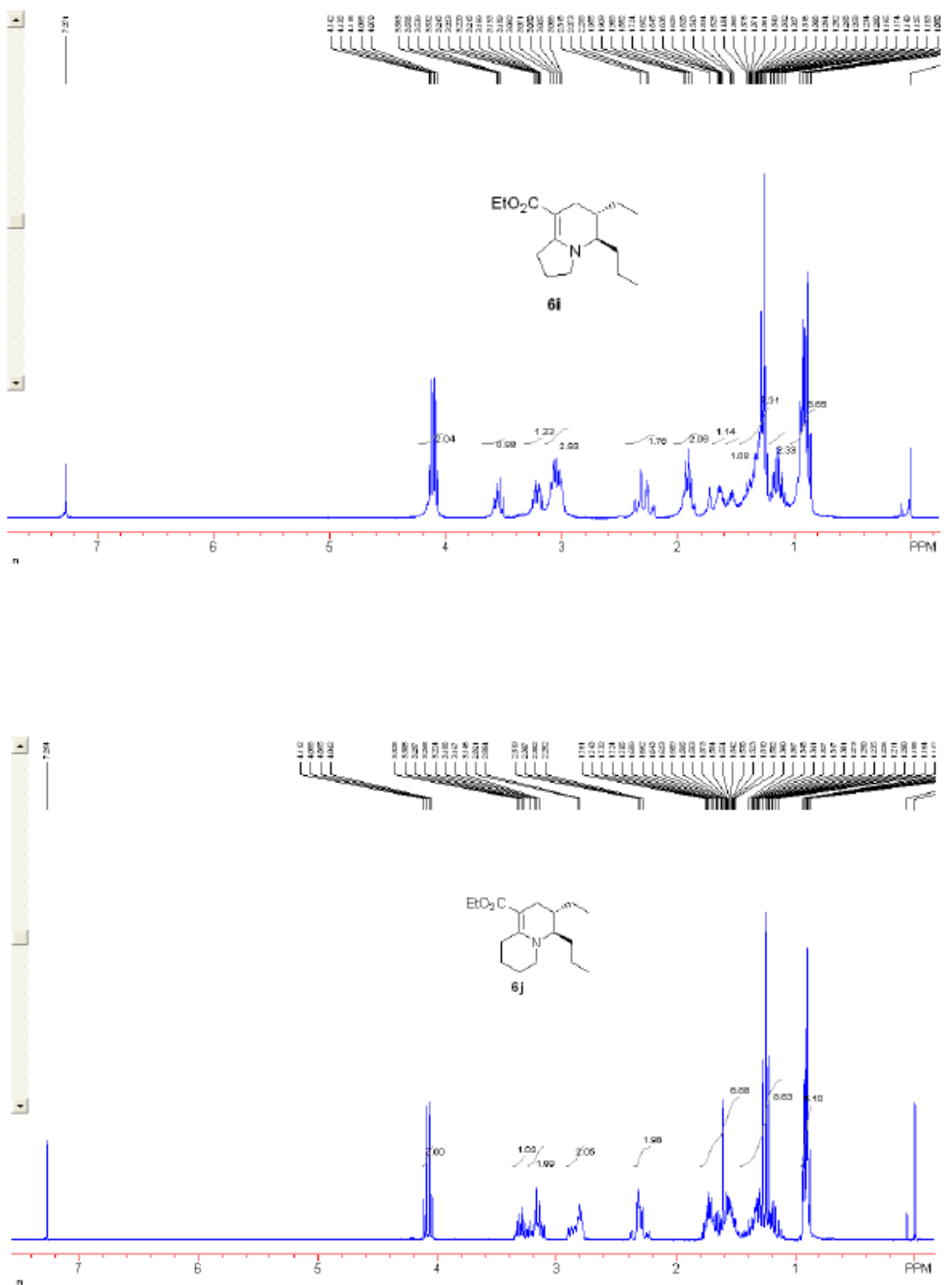

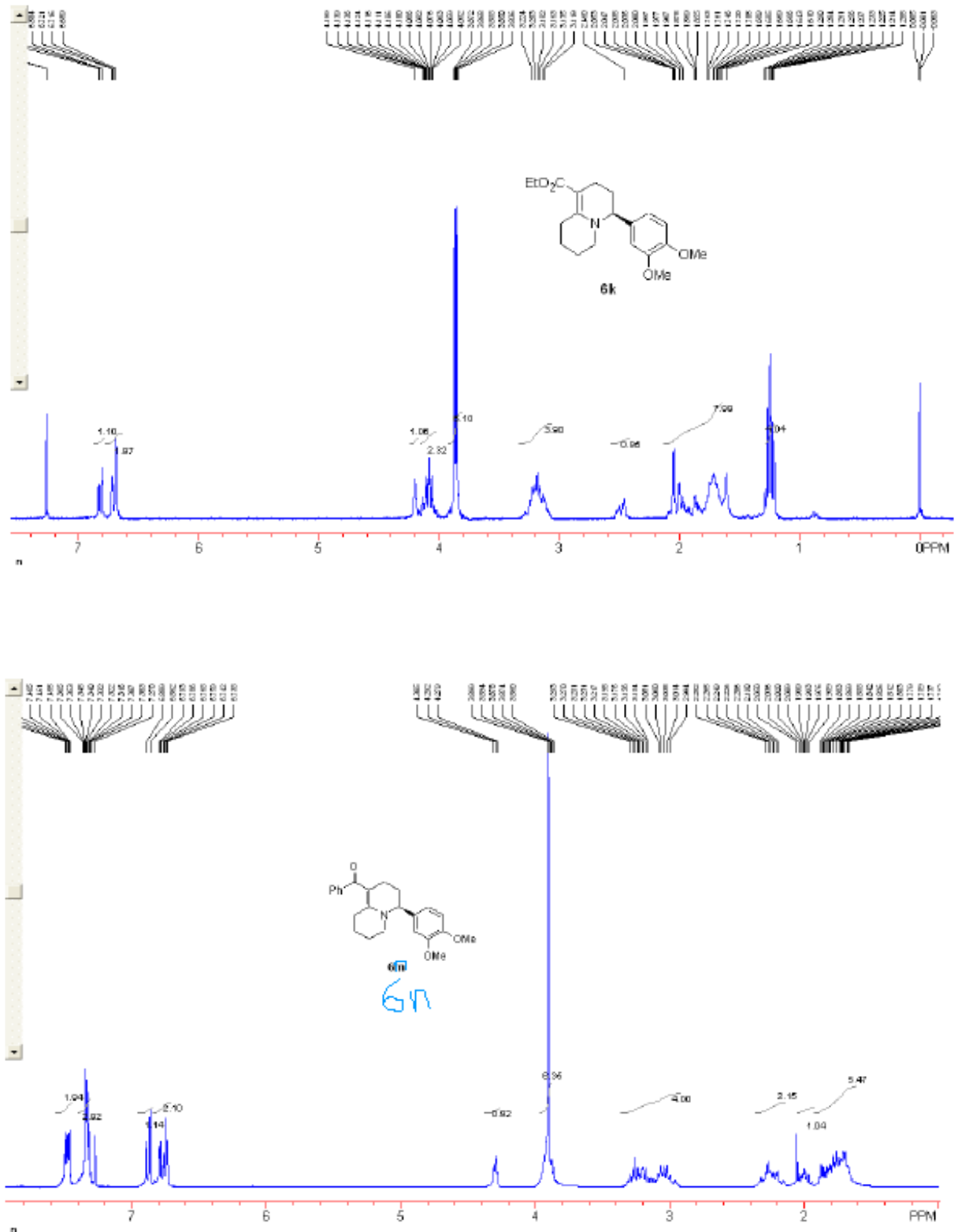

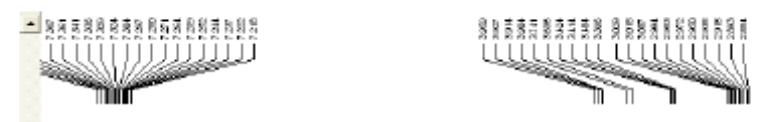

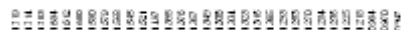
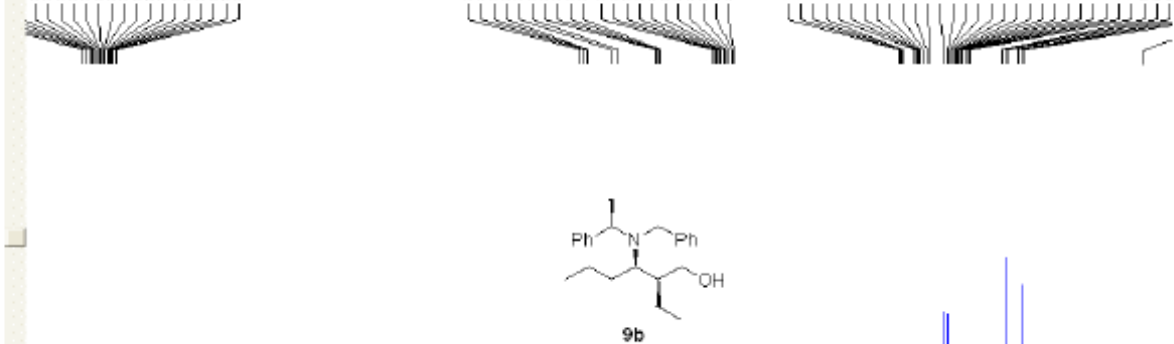

- .

$9 \mathrm{~b}$
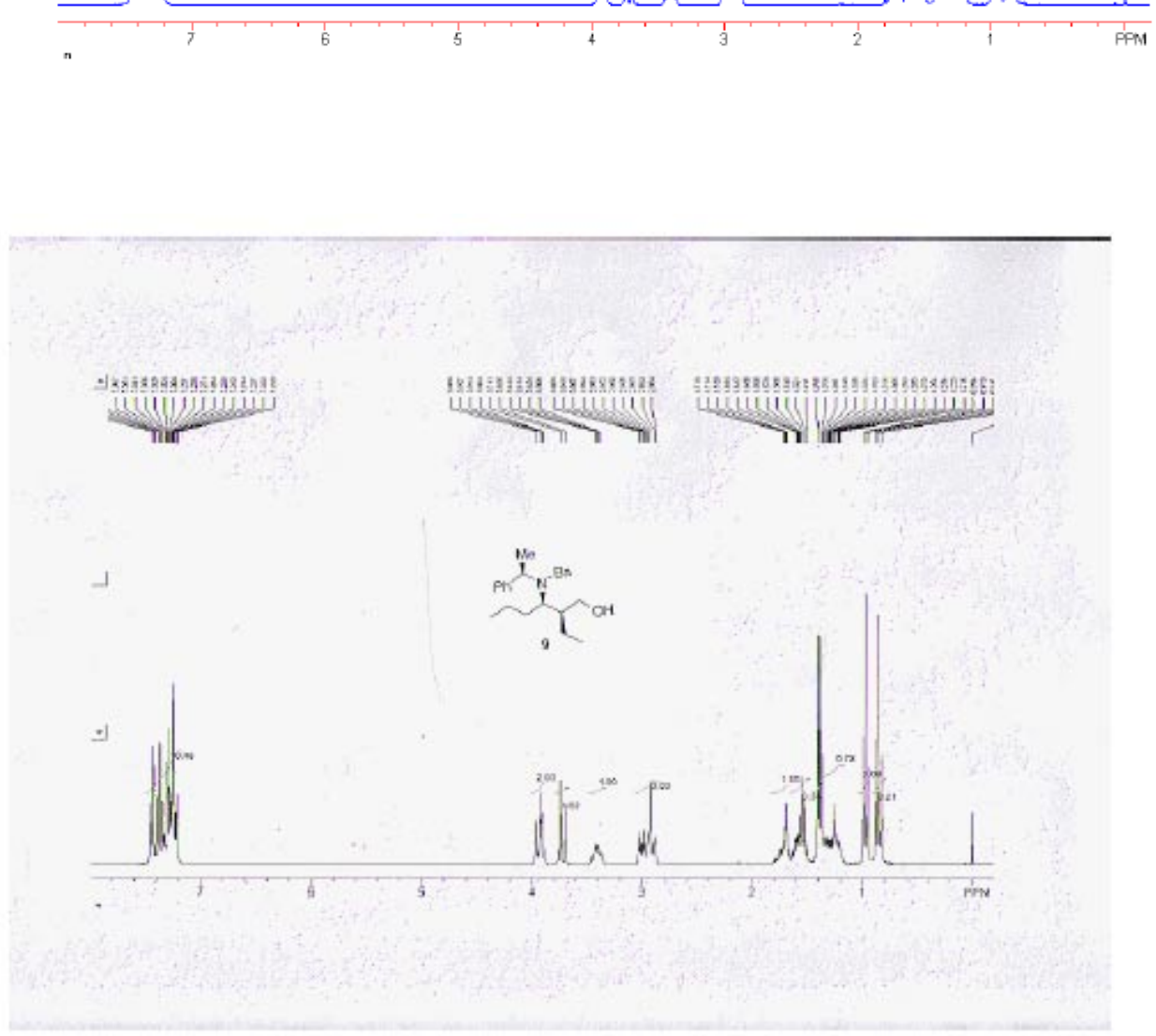

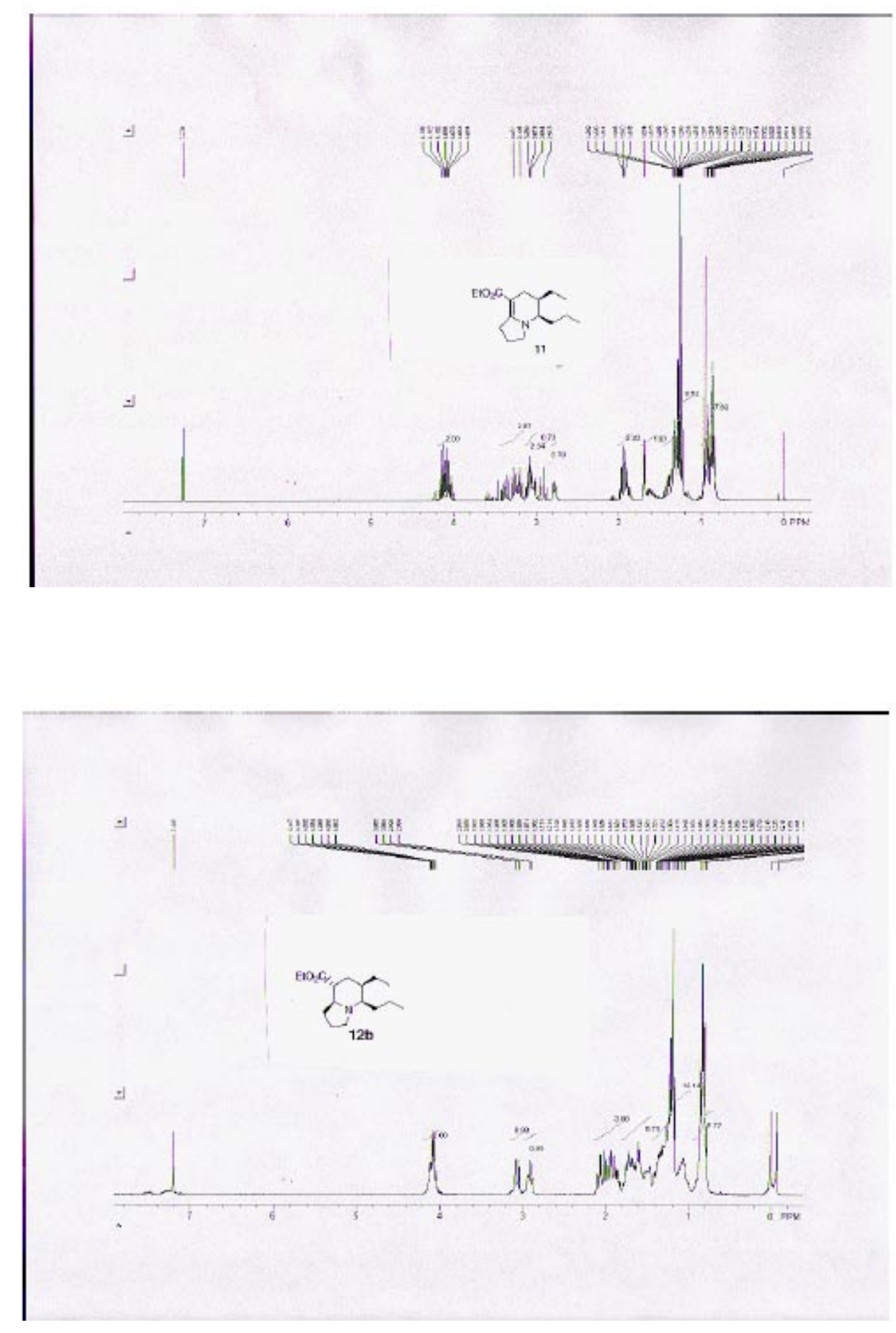

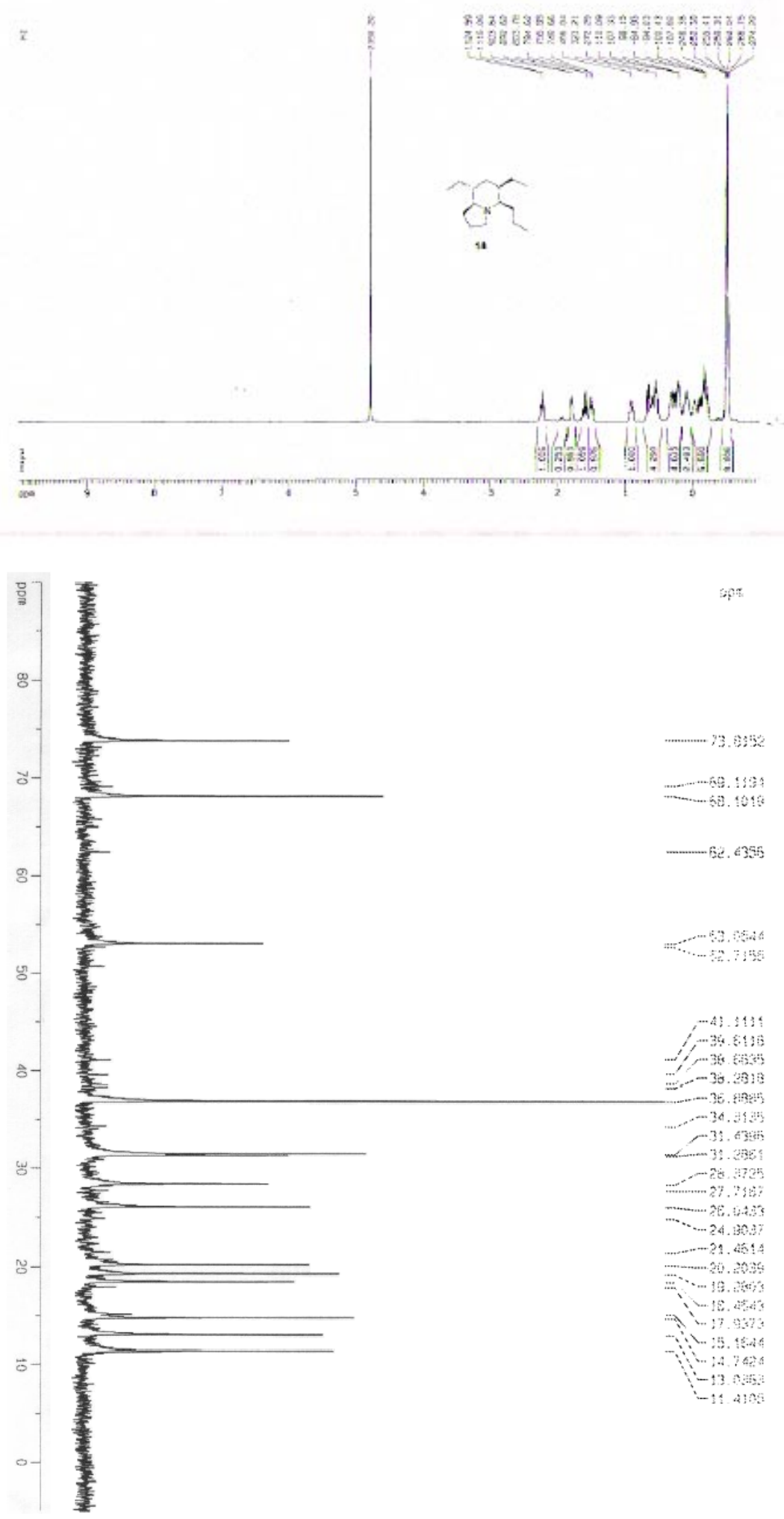